\title{
ON SOME GLOBAL AND LOCAL GEOMETRIC PROPERTIES OF CALDERÓN-LOZANOVSKIĬ SPACES
}

\author{
AGATA NARLOCH \\ Institute of Mathematics, Szczecin University, Wielkopolska 15, 70-451 Szczecin, Poland \\ E-mail: narloch@sus.univ.szczecin.pl
}

\begin{abstract}
Criteria for full $k$-rotundity $(k \in \mathbb{N}, k \geqslant 2)$ and uniform rotundity in every direction of Calderón-Lozanovskiı spaces are formulated. A characterization of $H_{\mu}$-points in these spaces is also given.
\end{abstract}

Introduction. First we introduce the notations and define the notions used in this paper. Let $(X,\|\|)$ be a real Banach space and $S(X), B(X)$ denote the unit sphere and the (closed) unit ball of the space $X$, respectively.

A Banach space $X$ is called fully $k$-rotund ( $k R$-space for short), where $k \in \mathbb{N}, k \geqslant 2$, if any sequence $\left(x_{n}\right)$ in $B(X)$ such that

$$
\left\|x_{n}^{(1)}+x_{n}^{(2)}+\cdots+x_{n}^{(k)}\right\| \rightarrow k
$$

for arbitrary subsequences $\left(x_{n}^{(1)}\right),\left(x_{n}^{(2)}\right), \ldots,\left(x_{n}^{(k)}\right)$ as $n \rightarrow \infty$, is a Cauchy sequence (see $[\mathrm{FG}])$. It is known that any $k R$-space is a $(k+1) R$-space $(k \geqslant 2)$.

A Banach space $X$ is said to be compactly fully $k$-rotund ( $C k R$-space for short) if every sequence $\left(x_{n}\right)$ in $B(X)$ satisfying

$$
\left\|x_{n}^{(1)}+x_{n}^{(2)}+\cdots+x_{n}^{(k)}\right\| \rightarrow k
$$

for any subsequences $\left(x_{n}^{(1)}\right),\left(x_{n}^{(2)}\right), \ldots,\left(x_{n}^{(k)}\right)$ as $n \rightarrow \infty$, is a relatively compact sequence. Compact full $k$-rotundity of a Banach space $X$ implies reflexivity (see [CHK]) and approximative compactness of the space $X$ (see $[\mathrm{HW}]$ ). A Banach space $X$ is fully $k$-rotund iff it is compactly fully $k$-rotund and rotund (see [CHK]).

We say that a Banach space $X$ is uniformly convex in every direction (URED-space for short) if for any $\varepsilon \in(0,1)$ and $z \in S(X)$ there exists $\delta(\varepsilon, z) \in(0,1)$ such that $\|(x+y) / 2\| \leqslant 1-\delta(\varepsilon, z)$ for any $x, y \in B(X)$ with $x-y=\varepsilon z$ or equivalently, if for any

2000 Mathematics Subject Classification: 46E30, 46B20, 46B30, 46C05.

Key words and phrases: Köthe space, Calderón-Lozanovskiı̆ space, Orlicz function, delta two type condition, fully $k$-rotundity, uniform rotundity in every direction, $H_{\mu}$-points.

The paper is in final form and no version of it will be published elsewhere. 
$\varepsilon \in(0,1)$ and $z \in S(X)$ there exists $\delta(\varepsilon, z) \in(0,1)$ such that inequality $\|y+\varepsilon z / 2\| \leqslant$ $1-\delta(\varepsilon, z)$ holds whenever $y \in B(X)$ and $\|y+\varepsilon z\| \leqslant 1$.

Recall that if a Banach space $X$ is $U R E D$, then it has normal structure and so it has the weak fixed point property (see [CCHS]).

Let $(T, \Sigma, \mu)$ be a complete and $\sigma$-finite measure space and $L^{0}=L^{0}(T, \Sigma, \mu)$ be the space of all (equivalence classes of) $\Sigma$-measurable real functions defined on $T$.

A Banach space $\left(E,\|\|_{E}\right)$ is said to be a Köthe space (see $\left.[\mathrm{KA}]\right)$ if $E \subset L^{0}$ and:

(i) for every $x \in L^{0}$ and $y \in E$ with $|x(t)| \leqslant|y(t)|$ for $\mu$-a.e. $t \in T$, we have $x \in E$ and $\|x\|_{E} \leqslant\|y\|_{E}$,

(ii) there is a function $x \in E$ such that $x(t)>0$ for any $t \in T$.

By $E^{+}$we denote the positive cone of $E$, that is, $E^{+}=\{x \in E: x \geqslant 0\}$.

A Köthe space $E$ is said to be uniformly monotone if for any $\varepsilon \in(0,1)$ there is $\delta(\varepsilon) \in(0,1)$ such that $\|x-y\|_{E} \leqslant 1-\delta(\varepsilon)$ whenever $0 \leqslant y \leqslant x,\|x\|_{E}=1$ and $\|y\|_{E} \geqslant \varepsilon$. For the conditions that are equivalent to this definition we refer to [HKM2].

We say that a Köthe space $E$ has the Fatou property $(E \in(F P)$ for short) if for any $x \in L^{0}$ and $\left(x_{n}\right)$ in $E^{+}$such that $x_{n} \uparrow|x| \mu$-a.e. and $\sup _{n}\left\|x_{n}\right\|_{E}<\infty$, we have $x \in E$ and $\left\|x_{n}\right\|_{E} \rightarrow\|x\|_{E}$ (see $[\mathrm{Bi}]$ and $[\mathrm{KA}]$ ).

A point $x \in E$ is said to have order continuous norm if for any sequence $\left(y_{n}\right)$ in $E$ such that $0 \leqslant y_{n} \leqslant|x|(n \in \mathbb{N})$ and $y_{n} \rightarrow 0 \mu$-a.e., we have $\left\|y_{n}\right\|_{E} \rightarrow 0$. If every point of $E$ has order continuous norm, then we say that the space $E$ is order continuous.

A point $x \in E$ is said an $H_{\mu}$-point if for any sequence $\left(x_{n}\right) \subset E$ such that $x_{n} \rightarrow x$ locally in measure and $\left\|x_{n}\right\|_{E} \rightarrow\|x\|_{E}$, we have $\left\|x_{n}-x\right\|_{E} \rightarrow 0$. If every point $x \in E$ is $H_{\mu}$-point, then we say that the space $E$ has $H_{\mu}$-property (see [HM]).

A function $\varphi:[0, \infty) \rightarrow[0, \infty]$ is said to be an Orlicz function if $\varphi$ is convex, vanishing and continuous at zero, left continuous on $(0, \infty)$ and not identically equal to zero (see $[\mathrm{Ch}],[\mathrm{KR}],[\mathrm{Lu}],[\mathrm{Ma}],[\mathrm{Mu}]$ and $[\mathrm{RR}])$. If the Orlicz function $\varphi$ vanishes only at zero, then we will write $\varphi>0$ and if $\varphi$ takes only values from $[0, \infty)$, then we will write $\varphi<\infty$.

Given a real Köthe space $E$ and an Orlicz function $\varphi$, we define on $L^{0}$ the convex modular

$$
\varrho_{\varphi}(x)= \begin{cases}\|\varphi \circ|x|\|_{E} & \text { if } \varphi \circ|x| \in E, \\ \infty & \text { otherwise. }\end{cases}
$$

The Calderón-Lozanovskiu space $E_{\varphi}$ generated by the couple $(E, \varphi)$ is defined as the set of those $x \in L^{0}$ such that $\varrho_{\varphi}(\lambda x)<+\infty$ for some $\lambda>0$. The norm in $E_{\varphi}$ is defined by

$$
\|x\|_{\varphi}=\inf \left\{\lambda>0: \varrho_{\varphi}(x / \lambda) \leqslant 1\right\}
$$

(see $[\mathrm{CHM}]$ and $[\mathrm{Ma}]$; cf. [Ca] and [Lo]). If $E$ has the Fatou property, then also $E_{\varphi}$ has this property, whence it follows that $E_{\varphi}$ is a Banach space. This class of Köthe spaces is a subclass of the more general class of Köthe spaces $\Psi(E, F)$ that are interpolation spaces between two Köthe spaces $E$ and $F$ over the same measure space generated by concave and homogeneous functions $\Psi: \mathbb{R}_{+} \times \mathbb{R}_{+} \rightarrow \mathbb{R}_{+}$. Köthe spaces constructed in such a way by Lozanovskiı (see [Lo]) are generalizations of the interpolation spaces constructed by Calderón (see [Ca]). 
In the remaining part of the paper we will assume that $E$ is a Köthe space with the Fatou property.

We say an Orlicz function $\varphi$ satisfies condition $\Delta_{2}(0)\left(\varphi \in \Delta_{2}(0)\right.$ for short) if there exist $K>0$ and $u_{0}>0$ such that $0<\varphi\left(u_{0}\right)$ and the inequality $\varphi(2 u) \leqslant K \varphi(u)$ holds for all $u \in\left[0, u_{0}\right]$.

We say a function $\varphi$ satisfies condition $\Delta_{2}(\infty)\left(\varphi \in \Delta_{2}(\infty)\right.$ for short) if there exist $K>0$ and $u_{0}>0$ such that $\varphi\left(u_{0}\right)<\infty$ and the inequality $\varphi(2 u) \leqslant K \varphi(u)$ holds for all $u \geqslant u_{0}$.

If there exists $K>0$ such that $\varphi(2 u) \leqslant K \varphi(u)$ for all $u \geqslant 0$, then we say that $\varphi$ satisfies condition $\Delta_{2}\left(\mathbb{R}_{+}\right) \quad\left(\varphi \in \Delta_{2}\left(\mathbb{R}_{+}\right)\right.$for short $)$.

For a Köthe space $E$ and an Orlicz function $\varphi$ we say that $\varphi$ satisfies condition $\Delta_{2}^{E}$ $\left(\varphi \in \Delta_{2}^{E}\right.$ for short) if:

1) $\varphi \in \Delta_{2}(0)$ whenever $E \hookrightarrow L^{\infty}$,

2) $\varphi \in \Delta_{2}(\infty)$ whenever $L^{\infty} \hookrightarrow E$,

3) $\varphi \in \Delta_{2}\left(\mathbb{R}_{+}\right)$whenever neither $L^{\infty} \hookrightarrow E$ nor $E \hookrightarrow L^{\infty}$

(see [HKM1]).

LEMMA 1. If $E_{\varphi}$ is a Calderón-Lozanovskiて space and $x \in E_{\varphi}$, then:

(i) if $\|x\|_{\varphi} \leqslant 1$, then $\varrho_{\varphi}(x) \leqslant\|x\|_{\varphi}$,

(ii) if $\|x\|_{\varphi}>1$, then $\varrho_{\varphi}(x) \geqslant\|x\|_{\varphi}$.

LEMma 2 (see [CHM], [FH1] and [FH2]). If $\varphi$ is an Orlicz function such that $\varphi<\infty$, $\varphi \in \Delta_{2}^{E}$ and $E$ is a Köthe space, then for any $x \in E_{\varphi}$ and any sequence $\left(x_{n}\right)$ in $E_{\varphi}$, we have:

(i) $\varrho_{\varphi}(x)=1$ whenever $\|x\|_{\varphi}=1$,

$\left(\right.$ ii) $\varrho_{\varphi}\left(x_{n}\right) \rightarrow 1$ whenever $\left\|x_{n}\right\|_{\varphi} \rightarrow 1$,

(iii) $\varrho_{\varphi}(\lambda x)<\infty$ for any $\lambda \geqslant 0$.

LEMMA 3 (see [CHM], [FH1] and [FH2]). Let $\varphi$ be an Orlicz function such that $\varphi>0$ and $\varphi \in \Delta_{2}^{E}$. Then for any sequence $\left(x_{n}\right)$ in the Calderón-Lozanovskiて space $E_{\varphi}$, we have $\left\|x_{n}\right\|_{\varphi} \rightarrow 0$ whenever $\varrho_{\varphi}\left(x_{n}\right) \rightarrow 0$.

REMARK 1. For any real numbers $a, b$ we have:

(i) if $a b \geqslant 0$, then $|a+b|=|a|+|b|$ and $|a-b|=|| a|-| b||$,

(ii) if $a b<0$, then $|a+b|=|| a|-| b||$ and $|a-b|=|a|+|b|$.

\section{Results}

Proposition 1. Let $E$ be a uniformly monotone Köthe space and $\varphi$ be an Orlicz function with $\varphi>0, \varphi<\infty$ and $\varphi \in \Delta_{2}^{E}$. If $E$ is fully $k$-rotund, then $E_{\varphi}$ is fully $k$-rotund $(k \geqslant 2)$.

Proof. Let $\left(x_{n}\right)$ be a sequence in $B\left(E_{\varphi}\right)$ such that

$$
\left\|x_{n}^{(1)}+x_{n}^{(2)}+\cdots+x_{n}^{(k)}\right\|_{\varphi} \rightarrow k \quad \text { as } n \rightarrow \infty
$$


for any subsequences $\left(x_{n}^{(1)}\right),\left(x_{n}^{(2)}\right), \ldots,\left(x_{n}^{(k)}\right)$ of $\left(x_{n}\right)$. By the assumptions that $\varphi \in \Delta_{2}^{E}$ and $\varphi<\infty$, we have $\varphi \circ\left|x_{n}\right| \in B(E)$ for any $n \in \mathbb{N}$ and

$$
\left\|\varphi \circ\left|\frac{x_{n}^{(1)}+x_{n}^{(2)}+\cdots+x_{n}^{(k)}}{k}\right|\right\|_{E} \rightarrow 1 \quad \text { as } n \rightarrow \infty .
$$

(see Lemmas 1 and 2) and therefore,

$$
\frac{1}{k}\left\|\varphi \circ\left|x_{n}^{(1)}\right|+\varphi \circ\left|x_{n}^{(2)}\right|+\cdots+\varphi \circ\left|x_{n}^{(k)}\right|\right\|_{E} \rightarrow 1 \quad \text { as } n \rightarrow \infty .
$$

The space $E$ is fully $k$-rotund, so (2) implies that $\left(\varphi \circ\left|x_{n}\right|\right)$ is a Cauchy sequence in $E$ that is

$$
\left\|\varphi \circ\left|x_{m}\right|-\varphi \circ\left|x_{l}\right|\right\|_{E} \rightarrow 0 \quad \text { as } m, l \rightarrow \infty .
$$

Using superadditivity of the function $\varphi$ we have

$$
\varphi \circ|| x_{m}|-| x_{l}|| \leqslant|\varphi \circ| x_{m}|-\varphi \circ| x_{l}||
$$

so the previous condition yields

$$
\varrho_{\varphi}\left(\left|x_{m}\right|-\left|x_{l}\right|\right)=\left\|\varphi \circ|| x_{m}|-| x_{l}||\right\|_{E} \rightarrow 0 \text { as } m, l \rightarrow \infty
$$

and, by $\varphi>0$ and $\varphi \in \Delta_{2}^{E}$, we get

$$
\left\|\left|x_{m}\right|-\left|x_{l}\right|\right\|_{\varphi} \rightarrow 0 \quad \text { as } m, l \rightarrow \infty
$$

(see Lemma 3). Observe that condition (1) yields

$$
\left\|x_{m}+x_{l}\right\|_{\varphi} \rightarrow 2 \quad \text { as } m, l \rightarrow \infty .
$$

Let us define for any $i, j \in \mathbb{N}$

$$
A_{i j}=\left\{t \in T: x_{i}(t) \cdot x_{j}(t)<0\right\} .
$$

We will show that

$$
\left\|\left(\left|x_{m}\right|+\left|x_{l}\right|-\left|x_{m}-x_{l}\right|\right) \chi_{A_{m l}}\right\|_{\varphi} \rightarrow 0 \quad \text { as } m, l \rightarrow \infty .
$$

If we suppose, on the contrary, that condition (5) is not true, then there exist increasing sequences $\left(m_{n}\right),\left(l_{n}\right)$ of natural numbers such that

$$
\left\|\left(\left|x_{m_{n}}\right|+\left|x_{l_{n}}\right|-\left|x_{m_{n}}-x_{l_{n}}\right|\right) \chi_{A_{m_{n} l_{n}}}\right\|_{\varphi} \geqslant \delta
$$

for some $\delta>0$ and any $n \in \mathbb{N}$. The uniform monotonicity of $E$ and the assumptions concerning $\varphi$ imply uniform monotonicity of $E_{\varphi}$ (see [CHM]). So, there exists $\eta>0$ such that $\left\|z_{n}+y_{n}\right\|_{\varphi} \geqslant 1+\eta$ for $n \in \mathbb{N}$ large enough, whenever $\left(z_{n}\right),\left(y_{n}\right) \subset E_{\varphi}^{+},\left\|z_{n}\right\| \rightarrow 1$ and $\left\|y_{n}\right\| \geqslant \frac{\delta}{2}(n \in \mathbb{N})$. Then, by (4) and Remark 1 , we have

$$
\begin{aligned}
& 1 \geqslant\left\|\frac{\left|x_{m_{n}}\right|+\left|x_{l_{n}}\right|}{2}\right\|_{\varphi}=\left\|\left(\frac{\left|x_{m_{n}}\right|+\left|x_{l_{n}}\right|}{2}\right) \chi_{T \backslash A_{m_{n} l_{n}}}+\left(\frac{\left|x_{m_{n}}\right|+\left|x_{l_{n}}\right|}{2}\right) \chi_{A_{m_{n} l_{n}}}\right\|_{\varphi} \\
& =\left\|\frac{\left|x_{m_{n}}+x_{l_{n}}\right|}{2} \chi_{T \backslash A_{m_{n} l_{n}}}+\left(\frac{\left|x_{m_{n}}\right|+\left|x_{l_{n}}\right|}{2}-\frac{\left|x_{m_{n}}+x_{l_{n}}\right|}{2}\right) \chi_{T \backslash A_{m_{n} l_{n}}}+\frac{\left|x_{m_{n}}+x_{l_{n}}\right|}{2} \chi_{A_{m_{n} l_{n}}}\right\|_{\varphi} \\
& =\left\|\frac{\left|x_{m_{n}}+x_{l_{n}}\right|}{2}+\left(\frac{\left|x_{m_{n}}\right|+\left|x_{l_{n}}\right|}{2}-\frac{\left|x_{m_{n}}+x_{l_{n}}\right|}{2}\right) \chi_{T \backslash A_{m_{n} l_{n}}}\right\|_{\varphi} \geqslant 1+\eta
\end{aligned}
$$

for $n \in \mathbb{N}$ large enough, a contradiction. This means that condition (5) holds. 
Using again Remark 1, we get the inequalities

$$
\begin{aligned}
&\left\|\left|x_{m}\right|-\left|x_{l}\right|\right\|_{\varphi}+\left\|\left(\left|x_{m}\right|+\left|x_{l}\right|\right) \chi_{A_{m l}}-\left|x_{m}+x_{l}\right| \chi_{A_{m l}}\right\|_{\varphi} \\
& \geqslant\left\||| x_{m}|-| x_{l}|| \chi_{T \backslash A_{m l}}+|| x_{m}|-| x_{l}|| \chi_{A_{m l}}+\left(\left|x_{m}\right|+\left|x_{l}\right|\right) \chi_{A_{m l}}-\left|x_{m}+x_{l}\right| \chi_{A_{m l}}\right\|_{\varphi} \\
&=\left\||| x_{m}|-| x_{l}|| \chi_{T \backslash A_{m l}}+\left(\left|x_{m}\right|+\left|x_{l}\right|\right) \chi_{A_{m l}}\right\|_{\varphi} \\
& \geqslant\left\|\left|x_{m}-x_{l}\right| \chi_{T \backslash A_{m l}}+\left|x_{m}-x_{l}\right| \chi_{A_{m l}}\right\|_{\varphi}=\left\|\left|x_{m}-x_{l}\right|\right\|_{\varphi}=\left\|x_{m}-x_{l}\right\|_{\varphi},
\end{aligned}
$$

which, by (3) and (5), yield

$$
\left\|x_{m}-x_{l}\right\|_{\varphi} \rightarrow 0 \quad \text { as } m, l \rightarrow \infty
$$

Analogously we can prove

Proposition 2. Let $E$ be a uniformly monotone Köthe space and $\varphi$ be an Orlicz function with $\varphi>0, \varphi<\infty$ and $\varphi \in \Delta_{2}^{E}$. If $E$ is compactly fully $k$-rotund, then $E_{\varphi}$ is compactly fully $k$-rotund $(k \geqslant 2)$.

REMARK 2. In the proof of Proposition 1 it is shown that for any Köthe space $E$ if the positive cone $E^{+}$is (compactly) fully $k$-rotund and $E$ is uniformly monotone, then $E$ is (compactly) fully $k$-rotund.

Proposition 3. If $E$ is a uniformly monotone Köthe space and $\varphi$ is a strictly convex Orlicz function satisfying the $\Delta_{2}^{E}$-condition, then $E_{\varphi}$ is a URED-space.

Proof. Let us fix $\varepsilon \in(0,1)$ and $z \in \varepsilon S\left(E_{\varphi}\right)$. Let $y \in B\left(E_{\varphi}\right)$ be such that $\|y+z\|_{\varphi} \leqslant 1$.

Since the space $E$ is uniformly monotone, $\varphi \in \Delta_{2}^{E}$ and $\varphi$ is strictly convex, so $E_{\varphi}$ is uniformly monotone (see $[\mathrm{CHM}]$ ) and in consequence, $E_{\varphi}$ is order continuous (see [Bi]). Therefore, we can find a measurable set $A$ with positive finite measure and a number $k>0$ such that

$$
1 / k \leqslant|z(t)| \leqslant k \text { for any } t \in A \text { and }\left\|z \chi_{A}\right\|_{\varphi} \geqslant 4 \varepsilon / 5 .
$$

Now we see that $\chi_{A} \in E$ and, since $\varphi>0$, we have $\varrho_{\varphi}\left(z \chi_{A}\right)>0$. Note that $\varrho_{\varphi}(y) \leqslant$ $\|y\|_{\varphi} \leqslant 1$ (see Lemma 1). In the following we will consider two cases separately.

$1^{\circ}$ Assume first that $A$ is not an atom. Let $U$ be an arbitrary subset of $A$ such that $0<\mu(U)<\mu(A)$. Since $E$ is a strictly monotone space (because it is uniformly monotone), we have

$$
\left\|\chi_{A}\right\|_{E}-\left\|\chi_{U}\right\|_{E}=: \delta_{1}>0 .
$$

Let us choose $l>0$ such that

$$
\varphi(l)\left\|\chi_{U}\right\|_{E}>1
$$

and define $B=\{t \in A:|y(t)| \leqslant l\}$. If we suppose that $\left\|\chi_{A \backslash B}\right\|_{E}>\left\|\chi_{U}\right\|_{E}$, then we have

$$
\varrho_{\varphi}(y) \geqslant \varrho_{\varphi}\left(y \chi_{A \backslash B}\right)=\left\|\varphi \circ|y| \chi_{A \backslash B}\right\|_{E} \geqslant \varphi(l)\left\|\chi_{A \backslash B}\right\|_{E}>\varphi(l)\left\|\chi_{U}\right\|_{E}>1,
$$

a contradiction. Therefore, $\left\|\chi_{A \backslash B}\right\|_{E} \leqslant\left\|\chi_{U}\right\|_{E}$, and, in consequence,

$$
\left\|\chi_{B}\right\|_{E}=\left\|\chi_{A}-\chi_{A \backslash B}\right\|_{E} \geqslant\left\|\chi_{A}\right\|_{E}-\left\|\chi_{A \backslash B}\right\|_{E} \geqslant\left\|\chi_{A}\right\|_{E}-\left\|\chi_{U}\right\|_{E}=\delta_{1}
$$

and

$$
\varrho_{\varphi}\left(z \chi_{B}\right)=\left\|\varphi \circ|z| \chi_{B}\right\|_{E} \geqslant \varphi(1 / k)\left\|\chi_{B}\right\|_{E} \geqslant \varphi(1 / k) \delta_{1}=: \delta_{2}>0
$$


$2^{\circ}$ Now we consider the case when $A$ is an atom. Let $l>0$ be such that

$$
\varphi(l)\left\|\chi_{A}\right\|_{E}>1 .
$$

Denote again $B=\{t \in A:|y(t)| \leqslant l\}$. If $\mu(A \backslash B)=\mu(A)$, then $\chi_{A}=\chi_{A \backslash B}$ and

$$
\varrho_{\varphi}(y) \geqslant \varrho_{\varphi}\left(y \chi_{A \backslash B}\right)=\left\|\varphi \circ|y| \chi_{A \backslash B}\right\|_{E}=\left\|\varphi \circ|y| \chi_{A}\right\|_{E} \geqslant \varphi(l)\left\|\chi_{A}\right\|_{E}>1 .
$$

But we have $\varrho_{\varphi}(y) \leqslant\|y\|_{\varphi} \leqslant 1$. Therefore, $\mu(A)=\mu(B)$ and $\varrho_{\varphi}\left(z \chi_{B}\right)=\varrho_{\varphi}\left(z \chi_{A}\right)>0$.

We have shown that there exist numbers $l, \delta>0$ (independent of $y$ ) such that, for the set $C=\{t \in A:|y(t)| \leqslant l\}$, we have

$$
\varrho_{\varphi}\left(z \chi_{C}\right) \geqslant \delta .
$$

Observe that

$$
\max \{|y(t)+z(t)|,|y(t)|\} \leqslant k+l
$$

and

$$
|(y(t)-z(t))-y(t)|=|z(t)| \geqslant 1 / k
$$

for $\mu$-a.e $t \in C$. So, by strict convexity of $\varphi$ there exists $p \in(0,1)$, depending on $k, l$ (i.e. depending on $z$ and $\varepsilon$ ) only, such that

$$
\varphi\left(\left|y(t)+\frac{1}{2} z(t)\right|\right) \leqslant \frac{1-p}{2}[\varphi(|y(t)+z(t)|)+\varphi(|y(t)|)]
$$

for $\mu$-a.a. $t \in C$. Therefore, we have

$$
\begin{aligned}
\varphi \circ \mid y+ & \frac{1}{2} z|=\varphi \circ| \frac{(y+z)+y}{2} \mid \\
& \leqslant \frac{1}{2} \varphi \circ|y+z| \chi_{T \backslash C}+\frac{1}{2} \varphi \circ|y| \chi_{T \backslash C}+\frac{1-p}{2}\left(\varphi \circ|y+z| \chi_{C}+\varphi \circ|y| \chi_{C}\right) \\
& \leqslant \frac{1}{2} \varphi \circ|y+z|+\frac{1}{2} \varphi \circ|y|-\frac{p}{2} \varphi \circ|y+z| \chi_{C}-\frac{p}{2} \varphi \circ|y| \chi_{C} .
\end{aligned}
$$

If we define $D=\left\{t \in C:|z(t)| \geqslant \frac{\delta}{4} \max \{|y(t)+z(t)|,|y(t)|\}\right\}$, then the inequality

$$
\left\|\varphi \circ|z| \chi_{C \backslash D}\right\|_{E} \leqslant \frac{\delta}{4}\left\|\varphi \circ|y+z| \chi_{C \backslash D}+\varphi \circ|y| \chi_{C \backslash D}\right\|_{E} \leqslant \frac{\delta}{2}
$$

holds and, in viev of (6), it gives

$$
\varrho_{\varphi}\left(z \chi_{D}\right) \geqslant \frac{\delta}{2}
$$

Assume now that $L_{\infty} \hookrightarrow E$. Since $\varphi \in \Delta_{2}^{E}$ and $\varphi>0$, there exist $v, K>0$ such that $\left\|\varphi(v) \chi_{T}\right\|_{E} \leqslant \delta / 4$ and $\varphi(2 u) \leqslant K \varphi(u)+\varphi(v)$ for any $u \in[0, \infty)$. Then we have

$$
\begin{aligned}
\frac{\delta}{2} \leqslant \varrho_{\varphi}\left(z \chi_{D}\right) & =\left\|\varphi \circ|z+y-y| \chi_{D}\right\|_{E} \leqslant\left\|\varphi \circ\left(\frac{1}{2}|2(y+z)|+\frac{1}{2}|2 y|\right) \chi_{D}\right\|_{E} \\
& \leqslant \frac{K}{2}\left\|\varphi \circ|y+z| \chi_{D}+\varphi \circ|y| \chi_{D}\right\|_{E}+\left\|\varphi(v) \chi_{T}\right\|_{E} \\
& \leqslant \frac{K}{2}\left\|\varphi \circ|y+z| \chi_{D}+\varphi \circ|y| \chi_{D}\right\|_{E}+\frac{\delta}{4}
\end{aligned}
$$


and, in consequence,

$$
\left\|\frac{p}{2} \varphi \circ|y+z| \chi_{D}+\frac{p}{2} \varphi \circ|y| \chi_{D}\right\|_{E} \geqslant \frac{\delta p}{4 K} .
$$

The uniform monotonicity of $E$ and conditions (7) and (9) imply that there exists $\eta>0$ (depending on $p, \delta$ and $K$ only) such that

$$
\varrho_{\varphi}\left(y+\frac{1}{2} z\right) \leqslant 1-\eta .
$$

Now, by the $\Delta_{2}^{E}$-condition for $\varphi$ there exists $\beta>0$, depending only on $\eta$, such that $\|x\|_{\varphi} \leqslant 1-\beta$ whenever $\varrho_{\varphi}(x) \leqslant 1-\eta$ for any $x \in E_{\varphi}$. Finally, we have

$$
\left\|y+\frac{1}{2} z\right\|_{\varphi} \leqslant 1-\beta .
$$

If $E \hookrightarrow L_{\infty}$, then $\|x\|_{\infty} \leqslant M$ for every $x \in B\left(E_{\varphi}\right)$ and some $M>0$. Since $\varphi \in \Delta_{2}^{E}$ and $\varphi$ takes on only finite values, there exists $K_{1}>0$ such that $\varphi(2 u) \leqslant K_{1} \varphi(u)$ for $u \in[0, M]$. Hence we have

$$
\frac{\delta}{2} \leqslant \varrho_{\varphi}\left(z \chi_{D}\right)=\left\|\varphi \circ|z+y-y| \chi_{D}\right\|_{E} \leqslant \frac{K_{1}}{2}\left\|\varphi \circ|y+z| \chi_{D}+\varphi \circ|y| \chi_{D}\right\|_{E}
$$

and

$$
\left\|\frac{p}{2} \varphi \circ|y+z| \chi_{D}+\frac{p}{2} \varphi \circ|y| \chi_{D}\right\|_{E} \geqslant \frac{\delta p}{2 K_{1}} .
$$

Now we deduce, as above, that there exists $\beta_{1}>0$ such that

$$
\|y+z / 2\|_{\varphi}<1-\beta_{1} .
$$

The remaining case when neither $L_{\infty} \hookrightarrow E$ nor $E \hookrightarrow L_{\infty}$ is analogous and even easier to handle because the $\Delta_{2}^{E}$-condition means in this case the $\Delta_{2}$-condition on the whole $\mathbb{R}_{+}$.

We say that $x \in E^{+}$is an $H_{\mu}^{+}$-point if for any sequence $\left(x_{n}\right)$ in $E^{+}$such that $x_{n} \stackrel{\mu \text { (loc) }}{\longrightarrow} x$ (locally in measure) and $\left\|x_{n}\right\|_{E} \rightarrow\|x\|_{E}$, we have $\left\|x_{n}-x\right\|_{E} \rightarrow 0$. If all points $x \in E^{+}$ are $H_{\mu}^{+}$-points, then we say that $E$ has $H_{\mu}^{+}$-property.

In Proposition 1 in $[\mathrm{HM}]$ it was proved that any order continuous Köthe space has the $H_{\mu}$-property if and only if it has the $H_{\mu}^{+}$-property. The next lemma is a local version of that proposition.

LEMMA 4. For any order continuous Köthe space $E$, a point $x \in E$ is an $H_{\mu}$-point if and only if $|x|$ is an $H_{\mu}^{+}$-point.

Proof. Sufficiency. We may assume that $x \in S(E)$. Let $\left(x_{n}\right)$ be an arbitrary sequence in $E$ such that

$$
x_{n} \stackrel{\mu(\mathrm{loc})}{\longrightarrow} x \quad \text { and } \quad\left\|x_{n}\right\|_{E} \rightarrow 1=\|x\|_{E} .
$$

We will show that $\left\|x_{n}-x\right\|_{E} \rightarrow 0$ (by the assumption that $|x|$ is an $H_{\mu}^{+}$-point). Observe that condition $x_{n} \stackrel{\mu \text { (loc) }}{\longrightarrow} x$ yields

$$
\left|x_{n}\right| \stackrel{\mu(\mathrm{loc})}{\longrightarrow}|x| .
$$


The point $|x|$ is an $H_{\mu}^{+}$-point, so we have

$$
\left\|\left|x_{n}\right|-|x|\right\|_{E} \rightarrow 0
$$

Therefore, there exist $y \in E^{+}$and an increasing sequence $\left(n_{k}\right)$ of natural numbers such that

$$
|| x_{n_{k}}|-| x|| \leqslant y
$$

for any $k \in \mathbb{N}$ (see Lemma 2 in $[\mathrm{KA}]$, p. 141). We may assume additionally that

$$
x_{n_{k}} \rightarrow x \mu \text {-a.e. on } T \text {. }
$$

Applying (11) we have the inequality

$$
\left|x_{n_{k}}-x\right| \leqslant y+2|x|
$$

for any $k \in \mathbb{N}$. Conditions (12) and (13) together with the order continuity of $E$ give

$$
\left\|x_{n_{k}}-x\right\|_{E} \rightarrow 0 \text {. }
$$

Now it remains to apply the double extract subsequence theorem to obtain

$$
\left\|x_{n}-x\right\|_{E} \rightarrow 0
$$

and to end the proof of sufficiency.

Necessity. Let $x$ be an $H_{\mu}$-point and $\left(x_{n}\right)$ be an arbitrary sequence in $E^{+}$such that $x_{n} \stackrel{\mu \text { (loc) }}{\longrightarrow}|x|$ and $\left\|x_{n}\right\|_{E} \rightarrow\|x\|_{E}$. Define $y_{n}:=f x_{n}(n \in \mathbb{N})$, where $f(t)=1$ if $x(t) \geqslant 0$ and $f(t)=-1$ if $x(t)<0(t \in T)$. Then, we have

$$
\left|y_{n}-x\right|=\left|f x_{n}-f\right| x||=\left|x_{n}-\right| x||
$$

for any $n \in \mathbb{N}$. Therefore, $y_{n} \stackrel{\mu \text { (loc) }}{\longrightarrow} x$. Moreover, $\left\|y_{n}\right\|_{E}=\left\|x_{n}\right\|_{E} \rightarrow\|x\|_{E}$. So, $\left\|y_{n}-x\right\|_{E} \rightarrow$ 0 and in consequence, $\left\|x_{n}-|x|\right\|_{E} \rightarrow 0$. This means that $|x|$ is an $H_{\mu}^{+}$-point.

Proposition 4. Let $E$ be an order continuous Köthe space and $\varphi$ be an Orlicz function with $\varphi>0, \varphi<\infty$ and $\varphi \in \Delta_{2}^{E}$. An element $x \in E_{\varphi}$ is an $H_{\mu}$-point if and only if $\varphi \circ|x|$ is an $H_{\mu}^{+}$-point in $E$.

Proof. Sufficiency. Without loss of generality, we may assume that $x \in S\left(E_{\varphi}\right)$. The order continuity of $E$ and conditions $\varphi>0$ and $\varphi \in \Delta_{2}^{E}$ imply that $E_{\varphi}$ is order continuous (see [FH1]). Therefore, by Lemma 4 , it suffices to show that $|x|$ is $H_{\mu}^{+}$-point. Let $\left(x_{n}\right)$ be an arbitrary sequence in $E_{\varphi}^{+}$such that

$$
x_{n} \stackrel{\mu \text { (loc) }}{\longrightarrow}|x| \text { and }\left\|x_{n}\right\|_{\varphi} \rightarrow 1 .
$$

So, in view of $\varphi \in \Delta_{2}^{E}$ and $\varphi<\infty$, we have

$$
\varrho_{\varphi}\left(x_{n}\right)=\left\|\varphi \circ x_{n}\right\|_{E} \rightarrow 1=\|\varphi \circ|x|\|_{E}
$$

(see Lemma 2). Condition (14) also yields

$$
\varphi \circ x_{n} \stackrel{\mu(\mathrm{loc})}{\longrightarrow} \varphi \circ|x| \text {. }
$$

Indeed, if $x_{n} \stackrel{\mu \text { (loc) }}{\longrightarrow}|x|$, then $x_{n_{k}} \rightarrow|x| \mu$-a.e. on $T$ for some increasing sequence $\left(n_{k}\right)$ of natural numbers. Hence, by continuity of the function $\varphi$, we get $\varphi \circ x_{n_{k}} \rightarrow \varphi \circ|x| \mu$-a.e. on 
$T$ which implies $\varphi \circ x_{n_{k}} \stackrel{\mu \text { (loc) }}{\longrightarrow} \varphi \circ|x|$. Applying the double extract subsequence theorem we obtain condition (15).

The element $\varphi \circ|x|$ is an $H_{\mu}^{+}$-point in $E$, so we obtain

$$
\left\|\varphi \circ x_{n}-\varphi \circ|x|\right\|_{E} \rightarrow 0
$$

and in consequence,

$$
\varrho_{\varphi}\left(x_{n}-|x|\right)=\left\|\varphi \circ\left|x_{n}-\right| x||\right\|_{E} \leqslant\left\|\varphi \circ x_{n}-\varphi \circ|x|\right\|_{E} \rightarrow 0,
$$

by superadditivity of $\varphi$ on $\mathbb{R}_{+}$. But $\varphi \in \Delta_{2}^{E}$ and $\varphi>0$, so

$$
\left\|x_{n}-x\right\|_{\varphi} \rightarrow 0
$$

(see Lemma 3), which means that $|x|$ is an $H_{\mu}^{+}$-point.

Necessity. We may assume that $x \in S\left(E_{\varphi}\right)$. Then, by $\varphi \in \Delta_{2}^{E}$ and $\varphi<\infty$, we have $\|\varphi \circ|x|\|_{E}=1$. Let us choose an arbitrary sequence $\left(y_{n}\right)$ in $E^{+}$such that $y_{n} \stackrel{\mu \text { (loc) }}{\longrightarrow} \varphi \circ|x|$ and $\left\|y_{n}\right\|_{E} \rightarrow 1$. The function $\varphi$ is an injection, so we can define $x_{n}:=\varphi^{-1} \circ y_{n}$ for all $n \in \mathbb{N}$. We have $x_{n} \in E_{\varphi}^{+}$and $\left\|x_{n}\right\|_{\varphi} \rightarrow 1$ because $\varrho_{\varphi}\left(x_{n}\right)=\left\|y_{n}\right\|_{E} \rightarrow 1$ (see Lemma 1). Moreover, condition $y_{n} \stackrel{\mu \text { (loc) }}{\longrightarrow} \varphi \circ|x|$, continuity of $\varphi^{-1}$ and the double extract subsequence theorem give

$$
\varphi^{-1} \circ y_{n}=x_{n} \stackrel{\mu(\mathrm{loc})}{\longrightarrow}|x|=\varphi^{-1} \circ \varphi \circ|x| .
$$

From the assumption that $x$ is an $H_{\mu}$-point in $E_{\varphi}$ we have that $|x|$ is an $H_{\mu}^{+}$-point in $E_{\varphi}$ (see Lemma 4), so

$$
\left\|x_{n}-|x|\right\|_{\varphi} \rightarrow 0
$$

By Lemma 2 in [KA] (page 141), there exist $z \in E_{\varphi}^{+}$and an increasing sequence $\left(n_{k}\right)$ of natural numbers such that

$$
\left|x_{n_{k}}-\right| x|| \leqslant z
$$

for all $k \in \mathbb{N}$. Then, we have

$$
x_{n_{k}}+|x| \leqslant z+2|x|(k \in \mathbb{N}) .
$$

The conditions $\varphi \in \Delta_{2}^{E}, \varphi<\infty$ and Lemma 2 yield $\|\varphi \circ(z+2|x|)\|_{E}<\infty$, which means, by $E \in(F P)$, that $\varphi \circ(z+2|x|) \in E$. Let $\left(n_{m}\right)$ be a subsequence of $\left(n_{k}\right)$ such that

$$
y_{n_{m}} \rightarrow \varphi \circ|x| \mu \text {-a.e. on } T \text {. }
$$

Now, by condition (16) and superadditivity of the function $\varphi$, we get

$$
\begin{aligned}
& y_{n_{m}}=\varphi \circ x_{n_{m}}=\varphi \circ\left|\left(x_{n_{m}}+|x|\right)-\right| x|| \leqslant|\varphi \circ| x_{n_{m}}+|x||-\varphi \circ| x|| \leqslant \\
& \varphi \circ\left|x_{n_{m}}+\right| x||+\varphi \circ|x| \leqslant \varphi \circ(z+2|x|)+\varphi \circ|x| .
\end{aligned}
$$

Therefore, the order continuity of $E$ and condition (17) imply that $\left\|y_{n_{m}}-\varphi \circ|x|\right\|_{E} \rightarrow 0$. Finally, applying the double extract subsequence theorem, we obtain $\left\|y_{n}-|x|\right\|_{E} \rightarrow 0$, which means that $\varphi \circ|x|$ is $H_{\mu}^{+}$-point in $E$. 
[Bi] G. Birkhoff, Lattice Theory, Amer. Math. Soc., Providence, 1967.

[Ca] A. P. Calderón, Intermediate spaces and interpolation the complex method, Studia Math. 24 (1964), 113-190.

[CCHS] S. Chen, Y. Cui, H. Hudzik and B. Sims, Geomertic properties related to the fixed point theory in some Banach function lattices, in: Handbook of Metric Fixed Point Theory, Kluwer, 2001, 339-389.

[CHK] Y. Cui, H. Hudzik and W. Kowalewski, On fully rotundity properties and approximative compatness in some Banach sequece spaces, Indian J. Pure Appl. Math. 34 (2003), 17-30.

[CHM] J. Cerda, H. Hudzik and M. Mastyło, On the geometry of some Calderón-Lozanovskiu spaces, Indag. Math. (N.S.) 6 (1995), 35-49.

[Ch] S. Chen, Geometry of Orlicz Spaces, Dissertationes Math. 356 (1996).

[FG] K. Fan and I. Glicksberg, Fully convex normed linear spaces, Proc. Nat. Acad. Sci. USA 41 (1955), 947-953.

[FH1] P. Foralewski and H. Hudzik, Some basic properties of generalized CalderónLozanovskiu spaces, Collectanea Math. 48 (1997), 523-538.

[FH2] P. Foralewski and H. Hudzik, On some geometrical and topological properties of generalized Calderón-Lozanovskǐ sequence spaces, Houston J. Math. 25 (1999), 523-542.

[HKM1] H. Hudzik, A. Kamińska and M. Mastyło, Geometric properties of some CalderónLozanovskiu spaces and Orlicz-Lorentz spaces, Houston J. Math. 23 (1996), 639-663.

[HKM2] H. Hudzik, A. Kamińska and M. Mastyło, Monotonicity and rotundity properties in Banach lattices, Rocky Mountain J. Math. 30 (2000), 933-950.

[HM] H. Hudzik and M. Mastyło, Strongly extreme points in Köthe-Bochner spaces, Rocky Mountain J. Math. 23 (1993), 899-909.

[HW] H. Hudzik and B. Wang, Approximative compactness in Orlicz spaces, J. Approx. Theory 95 (1998), 82-89.

[KA] L. V. Kantorovich and G. P. Akilov, Functional Analysis, Nauka, Moscow, 1989.

[KR] M. A. Krasnosel'skiı̌ and Ya. B. Rutickǐ̌, Convex Functions and Orlicz Spaces, Noordhoff, Groningen, 1961.

[Lo] G. Ya. Lozanovskiŭ, On some Banach lattices II, Sibirsk. Math. J. 12 (1971), 562-567.

[Lu] W. A. J. Luxemburg, Banach Function Spaces, Thesis, Delft, 1955.

[Ma] L. Maligranda, Orlicz Spaces and Interpolation, Seminars in Math. 5, Campinas, 1989.

[Mu] J. Musielak, Orlicz Spaces and Modular Spaces, Lecture Notes in Math. 1034, SpringerVerlag, 1983.

[RR] M. M. Rao and Z. D. Ren, Theory of Orlicz Spaces, Marcel Dekker, New York, 1991. 